\title{
Why are Events, Facts, and States of Affairs Different?
}

\author{
Ana Clara Polakof \\ Pontifícia Universidade Católica do Rio de Janeiro
}

DOI: $10.2478 /$ disp-2017-0029

BIBLID [0873-626X (2017) 44; pp. 99-122]

\begin{abstract}
This article claims that events, facts and states of affairs need to be differentiated. It takes as a starting point Chisholm's (1976) claim that only his ontology of states of affairs explains effectively thirteen sentences related to propositions and events. He does this by reducing propositions and events to states of affairs. We argue that our ontology also solves those problems. We defend a hierarchized Platonist ontology that has concrete entities (objects and events) and abstract entities (properties, facts and states of affairs). The distinctions we propose allow us to explain the pre-analytic data analyzed by Chisholm without reducing entities. We claim that our ontology provides a different way of explaining that data, and is, thus, promissory.
\end{abstract}

\section{Keywords}

Events, facts, states of affairs, ontology, Chisholm.

\section{Introduction}

Chisholm (1976) proposes that it is possible to analyze events and propositions as subtypes of states of affairs. He argues that his proposal is the best alternative to account for some sentences that seem to identify events and propositions. In this article, we argue that it is possible to account for those same sentences in an ontology that differentiates events, from facts, and from states of affairs. We defend a Platonist ontology which makes an ontological distinction between those categories. It is a hierarchized ontology that contains at level 0 concrete entities such as objects and events, and, from level 1 and up, it contains abstract entities such as properties, facts and states of affairs. We argue that, by postulating this hierarchy and these onto-

Disputatio, Vol. IX, No. 44, May 2017

Received: 14/01/2016 Revised: 18/07/2016 Accepted: 27/01/2017 
logical categories, we may respond to problems as recurrence, occurrence and perception without reducing events or facts to states of affairs. We will further argue that, not only do we answer effectively the problems posed by Chisholm (1976), we do it without reducing concrete entities to abstract ones.

For us to be able to defend these ideas, we first explain Chisholm's (1976) proposal. Specifically, we focus on how states of affairs are defined, how propositions and events are reduced to states of affairs, and explain the problems that Chisholm considers. Then, we explain our proposal, which assumes a Platonist ontology. We briefly explain which are the ontological categories we include, why we propose a hierarchized ontology, and explain what makes events, facts and states of affairs different. Next, we show how our proposal also solves the problems that Chisholm postulated without having to assume that events are concretized states of affairs. We focus on the problems related to the last seven examples that Chisholm (1976) presents, because they are supposed to involve events. Finally, we present some concluding remarks.

\section{Chisholm's (1976) states of affairs}

Chisholm defines states of affairs as "abstract entities which exist necessarily and which are such that some but not all of them occur, take place or obtain" (Chisholm 1976: 114). States of affairs do not depend on concrete, individual things. ${ }^{1}$ So, even if there were no concrete things, there would be states of affairs. He argues that states of affairs need to be accepted by someone, and compares his states of affairs with Frege's thoughts (Chisholm 1976: 117-118). He proposes as a non-trivial criterion of identity that for a state of affairs $p$ to be the same as a state of affairs $q, p$ entails $q$ and $q$ entails $p$. States of affairs exist necessarily (but do not necessarily occur), as do properties and relations. Given a property $G$, if it is exemplified, we have a state of affairs $p$ that obtains (for instance, there being horses), and if

\footnotetext{
${ }^{1}$ We respect here the use Chisholm proposes for thing: "...I will use 'thing' in a very broad sense. Whatever there is may be said, in this sense, to be a thing: hence properties and relations are themselves things, and so are physical objects, persons and shadows" (Chisholm 1976: 21).
} 
it is not exemplified we have a state of affairs that does not obtain (for instance, there being a round square). This implies assuming an extreme version of Platonism (Chisholm 1976: 119). We have, thus, an ontology that accepts that anything which may be accepted or thought of may be a state of affairs. In this sense, it is a very broad ontology of states of affairs. However, while he does accept states of affairs, he reduces events and propositions to states of affairs.

He argues that his ontology answers some interesting philosophical problems. These arise with thirteen true sentences which contain terms that are supposed to refer to events, propositions, and states of affairs. He argues that any theory that wants to deal with events, propositions, and states of affairs should explain to what the terms in those sentences refer to (they may be properties, individual things, times, or whatever may be referred to). He, then, defends that his alternative is the best one to answer those problems, which appear in the next sentences (Chisholm 1976: 115-116): ${ }^{2}$

(1) What Jones fears most is what Smith is trying to bring about.

(2) No proposition is both true and false.

(3) For all events, $p, q$, and $r$, the probability of $p \& q$ in relation to $r$ is the product of the probability of $p$ in relation to $r$ and the probability of $q$ in relation to $p \& r$.

(4) There being round squares is impossible.

(5) One way of explaining the occurrence of an event $e$ is to deduce $e$ from a conjunction consisting of certain laws of nature and certain events which are known to have some prior probability.

(6) For all events $p$ and $q$, if it is a law of nature (is physically necessary) that $p$ occurs, and if $p$ logically implies $q$, then it is

\footnotetext{
${ }^{2}$ The alternatives he considers are: (1) Kim's proposal of events as property exemplifications (see Kim 1976); (2) Davidson's proposal of events as concrete (see Davidson 1981a), and considers C. I. Lewis a variant of this view; (3) and a last alternative that he calls the exemplification view which says that events are exemplifications of states of affairs (cf. Chisholm 1976: 121). We will not consider any of these alternatives in this article, but they are opponents to Chisholm's proposal.
} 
a law of nature (is physically necessary) that $q$ occurs.

(7) Someone walking is now occurring in Chicago.

(8) Truman being elected President occurred prior to Eisenhower being elected President.

(9) An incumbent President being re-elected has occurred more than seventeen times.

(10)The storm came into being on Tuesday and was all over by Thursday.

(11) Smith being ill contributed causally to Brown being re-elected.

(12)Smith perceived Jones to be robbed.

(13) The third occurrence of Jones being robbed contributed causally to his illness.

He states that the first six items seem to commit us to states of affairs or propositions, and the last 7 to events. He argues that the first six may be said to correlate with propositions which are defined as abstract objects that exist in all possible worlds. However, since propositions are reducible to states of affairs, we may say that at least the first six pertain to sates of affairs (Chisholm 1976: 124). He, then, argues that the last seven may be said to correlate with events which may also be reduced to states of affairs (Chisholm 1976: 124). This view appears to be more problematic because time is involved, and since he defined states of affairs as abstract and eternal, it is possible to object that they may not be connected with places and times. To solve this problem, he proposes that states of affairs may be concretized. The idea is that in some cases, when we have an event, some properties are instantiated in concrete things that make the state of affairs concrete. For instance, we may have a state of affairs like Brutus killing Caesar that was concretized in $44 \mathrm{BC}$ by Brutus and Caesar and that occurred in a certain place. Thus, the idea of an event as a concretized state of affairs is introduced and events are also reduced to states of affairs. 
He explains cases such as (7) and (8) by using the following strategy: they both depend on places and times, and are concretized in those spaces and times, but they are both reducible to eternal states of affairs. In (9), we have a case that deals with recurring events: someone was elected twice. Chisholm argues that they are the same state of affairs concretized in different times, and perhaps places. The idea is that the state of affairs is eternal, but it occurs/is concretized in certain places and times. This seems to be, according to Chisholm (1976: 128-129), an advantage with respect to considering events as concrete because recurrence would have to make us accept "generic events". However, the idea of considering states of affairs as eternal seems to contradict (10). If they are eternal they should not come into being or pass away. He needs to paraphrase that sentence, and accommodate it to one that may state that the storm occurred from a time to another time and then ceased to occur. So, this would not be any problem for his proposal. Nevertheless, the name storm resulted problematic because, to be able to argue that it was a state of affairs, it was necessary to transform it, through paraphrases, to some gerundive (Chisholm 1976: 131). The analysis of (11) involves causality: when events occur, they may contribute causally to another event. Then, to reduce them to states of affairs, it has to be argued that both Smith being ill and Brown being re-elected were concretized when they occurred, and that, when that happened, the first may be said to have caused the other. Sentence (12) deals with a case in which perception seems to be directed to something concrete. However, states of affairs are not concrete. Chisholm argues that, in this case, it is not necessary to accept concrete events. According to him (Chisholm 1976: 135) we are perceiving individual things and states of affairs, not concrete events. (13) deals with particular occurrences. It involves a combination of the analyses of (11) and (12), and thus may be solved in more or less the same way. He, then, ends the analysis of these sentences as follows:

I believe, then, that the view of states of affairs here presupposed is adequate to the pre-analytic data here set forth. I know of no other philosophical theory which is, or even claims to be, adequate to all these data (Chisholm 1976: 136).

Chisholm's proposal is not unproblematic, and he latter abandoned 
the term states of affairs and replaced it with states (Chisholm 1990). However, he still characterizes events as states (Chisholm 1990: 416). So, the problem of the reducibility of events to states of affairs remains, and it should be further addressed. Davidson (1981b) deals specifically with the problem of recurrence. Davidson argues that the fact that we have recurring events does not commit us to eternal events (or to Chisholm's eternal states of affairs). Davidson defends that, even though Chisholm's account would be useful to explain recurrence, it is not sufficient to explain simple events and actions (Davidson 1981b: 169). ${ }^{3}$ A different problem related with this proposal is that, even though he did not have to commit to events as concrete particulars, he did have to commit to times. This is a commitment that he abandoned in 1990, which demonstrates that his commitment to times was in fact problematic (see Feldman and Feldman 2015, section 7). Then, reducing events to states of affairs is not unproblematic: it does not explain correctly individual events, and - to explain concretization - it needs an ontological commitment to time.

We claim, after explaining our proposal, that we may answer to those problems without using Ockham's razor, and by assuming a Platonist ontology that accepts events as concrete things, facts and states of affairs as abstract things, and does not force us to commit with time. ${ }^{4}$

\section{Events, facts and states of affairs}

We propose a Platonist ontology that has as its basic ontological categories objects, events, properties, facts and states of affairs. It is based on the work of Chateaubriand (2001 and 2005) which isat the same time - inspired by the works of Frege (1964), Russell (1908) and Whitehead and Russell (1910), among others. Since we

3 Joseph (2014: 110 and ff.) also deals with some of the problems of Chisholm's view while comparing it to Davidson's. However, he focuses on the form of sentences and causation. This is not directly related to the problem we are dealing with in this paper.

${ }^{4}$ From now on, we will use the term entity for those things that pertain to our ontology. 
accept that there are logical properties that lead to the paradoxes noted by Russell (1908), the ontology has to be hierarchized in levels and types. ${ }^{5}$ We have at the most basic level, that is level 0 , objects and events which, we argue, are concrete. Properties - understood here in a broad and universal sense that includes relations, functions, etc.- - appear for the first time at level 1 , and are abstract entities. They may appear in all levels higher than or equal to 1 because, in addition to non-logical properties such as being brown, we have logical properties such as existence that may appear in all levels starting from level $1 .{ }^{6}$ Facts appear from level 1 and up, and they involve the instantiation of a property in a concrete entity or entities. States of affairs appear from level 2 and up, and they involve the instantiation of a property in a property or properties that were not instantiated in any concrete entity/ies. Both facts and states of affairs are abstract entities. We have, thus, an ontology that has, as concrete entities, objects and events, and, as abstract entities, properties, facts and states of affairs.

We accept only those entities that are there, and our ontology has no place for (im)possible things. Alleged properties such as being a unicorn, or being a round square cannot be instantiated, and do not exist. Even though our Platonist ontology allows for universals that could — hypothetically — not be instantiated, they should be able to be instantiated. Being a unicorn, or being a round square cannot. There are no unicorns, there are no impossible objects, and there are no abstract objects in which those properties could be instantiated. ${ }^{7}$ Our properties, understood as Platonic universals, instantiate in lower level existing entities. Thus, a level 1 property, as being human, may be instantiated in a lower level 0 object, and a level 2 property, as

\footnotetext{
${ }^{5}$ Obviously, this is not the only alternative possible. However, it allows us to differentiate the complex entities efficiently.

${ }^{6}$ Existence is the property of existence, and not the existential quantifier.

${ }^{7}$ It may be argued that these properties are necessary to account for sentences that involve them, as suggested by the reviewer. However, we are convinced that natural languages allow us to speak about fictional and non-existing/impossible things because they do not always reflect reality. Thus, the fact that I may speak about Sherlock Holmes does not commit myself to the existence of Sherlock Holmes. This argument was defended by Chateaubriand (2001).
} 
existence2, may be instantiated in a lower level entity (it may be a property or a fact). Logical properties are more significant in the hierarchy, and may be instantiated both in non-logical entities (such as objects, events, facts, non-logical properties and non-logical states of affairs) and in logical entities (such as other logical properties, or logical states of affairs). The hierarchy we assume, to account for the logical properties, is flexible and cumulative — as is Chateaubriand's (see Sautter 2010). This means that the logical properties may have a variable arity - they may combine with a varying number of arguments - ; they may appear in all levels from level 1 of the hierarchy; and combine with different kinds of entities. Let's exemplify with the logical property diversity. Since it is a flexible hierarchy, it may be combined with two objects (Diversity1 may combine with a dog and a cat), with three objects (Diversityl may combine with a dog, a cat, and $a$ table), and so on. Since it is cumulative, Diversity may appear in different levels - we may have diversity at level 2 , at level 3 , and up to infinity because the hierarchy does not have an upper bound. ${ }^{8}$ It may also combine with different entities — we may have, for instance, diversity2 that combines with a level 0 object, a level 0 event, a level 1 property, and a level 1 fact (Diversity2 may combine with a dog, a sunset, being brown, and <being brown, a $\operatorname{dog}>$ ). ${ }^{9}$

The hierarchy is important to the distinction we propose between events, facts and states of affairs. These are all complex entities, but may be differentiated: the first one is concrete, while the other two are abstract. Since the first one is concrete, it appears only in level 0. Since the others are abstract, they appear for the first time at level 1 and level 2 respectively. We claim that facts need to be differentiated from states of affairs, because the first ones involve concrete entities, and the second ones do not. ${ }^{10}$ We claim that, since facts involve level 0 entities they are temporal: they exist only when the concrete

${ }^{8}$ Diversity1, Diversity2, Diversity3, and so on are different - though similarproperties.

${ }^{9}$ This last argument is a fact $<$ being brown, a $\operatorname{dog}>$. It may be read in the following way: the first appearance is the property, in this case being brown, that is being instantiated in an entity, in this case the object dog.

${ }^{10}$ We talk about concrete entities because facts may involve objects and/or events. 
entity/ies involved exist, and they cease to exist when they cease to exist (and did not exist before that). ${ }^{11}$ On the other hand, since states of affairs only involve properties - and properties are atemporal-, they are atemporal and eternal. Facts result from the instantiation of a property in a concrete entity or entities, and thus, may appear at level $1 .{ }^{12}$ However, they are not restricted to level 1 alone. Whenever we have level 0 entities involved (they may be directly involved, as in a level 1 fact, or indirectly involved, as in a higher level fact), we will be in front of a fact. Thus, we are as much in front of a fact in the case of $<$ being brown, a dog $>$ as in the case of $<$ Existence $2,<$ being brown, $a \operatorname{dog}>>$. States of affairs involve the instantiation of a property in a property or properties, and thus, may appear for the first time at level 2. They may also appear at higher levels, and the only restriction that they have is that they may not involve objects and/ or events. Thus, the properties in which the property is instantiated may not have been instantiated in a concrete entity (but may have been instantiated in another property/ies or even another state of affairs). States of affairs, in contrast with facts which are always nonlogical, may be non-logical and logical. We have a non-logical state of affairs when a non-logical property is involved, as in the level 2 state of affairs <Universal Subordination, being human, being mortal>. We have a logical state of affairs when only logical properties are involved, as in the level 2 state of affairs $<$ Existence2, Diversity $1>$, and the level 3 state of affairs $<$ Diversity3, Identity2, Diversityl, $<$ Existence2, Diversityl $>>$. These characteristics show both that facts are different from states of affairs, and that they may not be reduced to another: one depends upon the concrete, and the other does not. Facts may

${ }^{11}$ Note that this existence is very different from the one used by Chisholm (1976: 119) who claims that entities may exist without them occurring. Our existence, understood here as a property (not as the existential quantifier, as mentioned in a previous footnote), implies that the entity which exists occurs. In the case of concrete entities, it may be understood "as the property of having a location in space time" (Zalta 1993: 404).

${ }^{12}$ Both facts and states of affairs pertain to the same level of the property that is being instantiated. That is why if a level 1 property is being instantiated in a level 0 object, we will have a level 1 fact; and if a level 2 property is instantiated in a level 1 property, we will have a level 2 state of affairs, as we will show. In this, we follow Chateaubriand (2001). 
be taken as more fundamental than states of affairs. A naturalist may accept facts, but never states of affairs. An ostrich nominalist could find our approach to facts interesting, and may even work with it, without accepting the more general states of affairs.

Even if we may be content with these definitions, there are several questions (noted by the reviewer) that arise with regards to our proposal: What does it mean to say that facts are temporal? How are they not spatial? If facts involve the instantiation of a property in a concrete entity, how are they abstract? What does it mean to say that facts come or cease to exist? A better understanding of the distinction between abstract and concrete is necessary to answer these questions. We propose that the distinction between abstract and concrete may be reduced to two characteristics: only concrete entities may be delimited spatio-temporally and may be involved in causality. ${ }^{13}$ This may be labelled a naïve distinction (Honderich 2005: 3), but it is a distinction that may be defended (see Lowe 2001: 52-53). Thus, it is possible to argue that facts are temporal, while still being abstract. They are temporal because they depend on the temporality of the object in which the property is instantiated. They are not spatial because, even though the fact results from the instantiation of a property in an object, the property is not in the object. If it were, we would be talking about tropes or ways of being of the object, and we are not. Even though we may state that the wall is white, and we surely see/perceive whether the wall is white or not, we defend that - in those cases - we are in front of concrete states (events) and not facts. Facts may not be seen, they may not be perceived, because they are abstract. They exist, but since they depend on the existence of the object, they come to exist when the object exists, and cease to exist when it ceases to exist. It may be said that objects possess properties, and that possession may said to be concrete (see Lowe 2006: 75). But facts are not possessed properties; facts are instantiated properties in concrete entities. This is why they may be said to be abstract, and they may be said to come to exist (which is different from coming into being, which is something that concrete entities

\footnotetext{
${ }^{13}$ Note that we are talking about space-time, and not about space and/or time as Lowe (2001: 155) does. We consider that for something to be concrete it must be in space-time (not only in space or only in time).
} 
may do, but not abstract ones).

Having explained what facts and states of affairs are, we need to explain what events are, and why they may not be reduced to facts or states of affairs. We defend that events are concrete entities, and particulars (in accordance with Davidson 1981a). They are spatiotemporally continuous entities (against Lemmon 1967: 99). That is, if some alleged event may stop for a time, it is not a single event. A battle that stops for the soldiers to have tea (example given by Lemmon) would be, according to him, a single event. We defend that it is not; that, if the soldiers stopped to have tea, the battle may not be a single event. If I walk, then, sit on a bench, and then start to walk again, there are three different events. Thus, the spatio-temporally continuous condition needs to be met for something to be an event. Events involve objects in happenings - term introduced to avoid using less neutral terms such as action, activity, change or movement. Events may not be defined as actions, since there are events without agents (like the rain, or the falling of a rock). There are events which do not involve activities (like my sitting); and that term has a strict linguistic use that involves the lexical aspect of verbs (like swimming) we wish to avoid. There are changes that are not events (Cambridge events, such as becoming a widow), and it may even be argued that non-changes are events (Lewis 1986: 261). Philosophers as Dretske (1967: 492) have argued that events do not move. And, states (Kim 1976: 310) could be considered a subtype of events, and they do not involve movement. Thus, events should not be defined as objects in movement. The new term, happenings, should be preferred. Happening is an epistemic notion that allows us to explain how the event is differentiated from the object. A happening may be conceived as involving actions (my writing of this article), movement (my bitting of my lip), changes ( $m y$ growing old), activities (my swimming), or as not involving any of them (my sitting). It is a way we have to classify events. This does not mean that actions are hidden behind happenings, since we can have events that are actions. When we talk of an event as an entity that involves an object in a happening, we are understanding that an event is a complex entity that involves an object doing/performing/making/ receiving something. Stating that does not say anything with respect to the type of event we are in front (if it is an action, or a change, or an activity, etc.). It just allows us to know that we are in front of an 
event and not of an object. That is, it allows us to distinguish objects from events. And lastly (and perhaps the most polemic point so far), events are independent of our (collective) knowledge, our culture and our society. Something idiosyncratic may be understood as an event by a given society, and not by another. A traditional Indian wedding lasts around three days, and it is conceived in that culture as a single event. However, if an outsider did not know anything about that convention, he could take it as three different events. The idea is that, once (collective) knowledge, society and culture are involved, we are not in front of ontological events. They could be epistemic/ cultural/social events, but not ontological events. When these conditions are taken into account, it is possible to explain why something as my writing right now is an event, but something as a party is not: the first meets the conditions enumerated, while the second does not. Of course, it could be argued (as the reviewer noted) that it is difficult to get clear-cut distinctions with regards to what is cultural and what is not. The idea is that, even though it could be argued that many events depend on epistemic subjects participating in them, the event has to be independent of our (collective) knowledge/culture/society. It may not involve plural agents/participants. So, even though something as my writing obviously involves knowledge, since I'm the sole agent of the event, it may be considered an event. Something as a party, or a soccer match, or a game of chess, involve multiple agents, social rules, conventions, and may be spatio-temporally discontinuous. This allows us to continue to argue that they are not events.

The possibility of having two co-occurring events needs to be addressed. We want to defend that they must be different, and that this follows from the conditions we listed before. Imagine that my biting of my lip now is an event that is co-occurring with my writing of this article. ${ }^{14}$ They are different events, even if they involve the same object. They are different because they involve different happenings of the same object; and they do not occupy the same space-time (they involve different parts of my body). Imagine, now, that I stop biting

${ }^{14}$ We will try not to enter into problematic assumptions of what kinds of expressions may refer to an event. We will use perfect nominals (as in Vendler 1967) to try to avoid problems associated with the expressions used. 
my lip and continue to write. Then, the time they occupy differs, and one of the events no longer exists. Both events were spatio-temporally continuous: I had not stopped biting my lip (until I stopped), neither had I stopped writing. When I stopped biting my lip, the event ended. Thus, we would have two events that co-occurred for a while, but then did not. Now, if I stop writing, and start again, we would have different events: one that does no longer exist, and another that exists in this moment. It does not matter that we are in front of the same object in the same happening. If the condition of the continuity in space-time is violated, we are dealing with two different events. None of these events depend on how we socially conceive them: there is no one writing of this article and no one biting of my lip, there were in fact many (I started and stopped uncountable times), and now there is only one event. How we refer to those events has nothing to do with the fact that my writing of this article is an event right now. We may talk about all my writings of this article as one event, as we may talk about a set of objects as one object, but that would be culturally conceived as an event in much the same way as a set is conceived as an object. Neither the writings of my article nor the set of level 0 objects constitute a level 0 entity. Thus, we could not be in front of a single event. If anything, we could be in front of a level 1 fact which involves the combination (a set of) multiple events. The idea of only one writing depends on how we conceive the world, but it does not imply that we are in front of a single event.

The goal of this article was to provide an alternative to Chisholm's proposal that could deal with the problems he presented, while maintaining an irreducibility of events, facts and states of affairs. ${ }^{15} \mathrm{We}$ defended that facts are irreducible to states of affairs, because they involve concrete entities, and are temporal. On the other hand, states of affairs involve abstract entities alone, and the concrete should not be reduced to the abstract. Events cannot be reduced to logical states of affairs, because events are not logical, and they do not involve any

\footnotetext{
${ }^{15}$ A different alternative would be to reduce everything to objects and ways of the objects being (Martin and Heil 1999). That could simplify the task. However, we want to defend that events, facts, and states of affairs are basic ontological categories. Thus, this is not an approach we take into account.
} 
logical entity. However, since we accept that there are non-logical states of affairs as <Universal Subordination, being human, being mortal $>$, we need to explain why it is not possible to reduce events to those general non-logical states of affairs. We argue that this reduction is not possible even with states of affairs like <Universal Subordination, being a falling, being an event $>$ which seem to involve events. These are states of affairs that involve properties that may be instantiated only in events. However, in those cases, we are in front of uninstantiated properties. Thus, they are independent of the concrete event that may or may not be occurring in this moment. ${ }^{16}$ Imagine that there is no falling occurring right now, the state of affairs is still there. For the event to be reducible to a state of affairs, we would have to take a Chisholmian approach to events, and this is not what we want to defend: there is no concretization of states of affairs since they do not involve the concrete. We argue that, if events were reducible to states of affairs, we should argue that states of affairs like <Universal Subordination, being human, being mortal> make objects reducible to states of affairs. And, we do not want to reduce the concrete to the abstract. Facts, on the other hand, do involve concrete entities. Does this mean that we may reduce events to facts? Our facts are temporal, and come to existence when the concrete entity comes to exist. Then, if we wanted to reduce events to facts, we should redefine our facts, since they would have to be prior to our events. However, events - if anything - are prior to facts. If an event does not come into being, a fact does not come to exit. Then, a fact that involves an event, such as <being slow, my writing of this article $>$, depends on the writing of my article. If I had not started to write this article, there could not be a fact that involved the instantiation of the non-logical property being slow in my writing of the article. Then, the coming into being of the event is prior to the coming into existence of the fact. ${ }^{17}$

\footnotetext{
${ }^{16}$ In this, they are similar to Chisholm's states of affairs. However, Chisholm's states of affairs seem to involve the idea that there are abstract objects (such as Brutus) that pertain to the state of affairs, and then are concretized in a particular event. As we mentioned previously, our ontology has no place for abstract objects. Thus, our proposal is different to Chisholm's even in this (since our states of affairs do not involve objects of any kind, and they cannot be concretized).

${ }^{17}$ Note that while concrete entities come into being and into existence at the same time, abstract entities may only come into existence for they are not in
} 
The irreducibility of events to facts is not very different from the irreducibility of objects to facts. A fact that involves an object, such as $<$ Existencel, my $\operatorname{dog}>$, does not make the object reducible to the fact. Again, we do not want to reduce the concrete to the abstract. Even though we argued that facts are temporal, they are abstract, they are not in space-time and they do not stand in causal relations. The argument would, then, follow the same path that was used for states of affairs: unless we are willing to reduce objects to facts, it does not seem plausible to reduce events to facts. And it may be obvious by now, that we are not willing to reduce the concrete to the abstract.

\section{Solving Chisholm's (1976) problems}

In this section, we want to show that our proposal answers the problems posed by Chisholm (1976) as effectively as his proposal. The first six sentences, according to Chisholm, refer to propositions which may be reduced to states of affairs. They are not analyzed in this article, because we are not taking propositions into account. ${ }^{18}$ However, we agree with him in that the sentences (1) to (6) could be propositions. ${ }^{19}$ In the rest of the section, we will focus in the last seven cases which are renumbered from (14) to (20):

(14) Someone walking is now occurring in Chicago.

(15) Truman being elected President occurred prior to Eisenhower being elected President.

space-time, as mentioned.

${ }^{18}$ We could analyze the first six ones, but they are very problematic. We do not think that they all refer or identify entities (besides propositions). Of course, in Chisholm's very broad sense of states of affairs, this is not a problem because anything that may be accepted by someone may be said to be a sate of affairs. However, in an ontology like the one we propose, everything needs to be considered carefully because the entities are independent of their being accepted or not. This is why we do not analyze these sentences in this article: because they do not necessarily correspond to any of the entities we proposed.

${ }^{19}$ We think that the ontological status, if any, of propositions is controversial. We, for instance, do not consider propositions to correspond to an ontological category. Where they should stand should be further researched. 
(16)An incumbent President being re-elected has occurred more than seventeen times.

(17)The storm came into being on Tuesday and was all over by Thursday.

(18) Smith being ill contributed causally to Brown being re-elected.

(19) Smith perceived Jones to be robbed.

(20) The third occurrence of Jones being robbed contributed causally to his illness.

These are the cases that, according to Chisholm (1976), involve events. We argue that the difference we propose between events, properties, facts and states of affairs may also resolve the problems posed by Chisholm. We propose that the first case, which stands for a true statement for there is someone walking now in Chicago, tries to identify an event. However, since we are not in Chicago right now, we cannot uniquely identify the location of the event nor the person involved in it; but since we know that there is someone walking we try to identify that event. And even if we cannot do it precisely (since it is indefinite), the truth of that statement and the relation of that statement to some event in the world cannot be questioned. This sentence, then, can be explained by our proposal. The second case, (15), does not identify any event in our proposal. Since events come into being when they occur, and the nominals of that sentence involve past events, there is no event to be identified in this moment. They were events at some moment, but they are not now. We may talk about them as historical events, but there is no entity to be identified by (15). Our ontology does not have historical entities (be them objects or events or facts), since this would involve accepting some sort of atemporal objects, events and/or facts. However, this does not mean that we cannot talk about nonexistent entities. As Parsons (1999) showed, we do this all the time, but this does not mean that we have to commit ourselves to the existence of these events.

The third case, (16), involves recurrence. According to Chisholm, it may not be explained by a theory that assumes events as concrete - unless generic events are accepted. It is true that, since we defend 
that events are concrete, we cannot argue that they recur (in the same way that no one would argue that objects recur). However, we do not need generic events. In our ontology, generality is related to properties and states of affairs alone. To avoid giving the same argument defended in (15) (that they are not events), (16) needs to be paraphrased to an example that could explain recurrence by our theory. That is, we have to provide a sentence that - once usedcould refer to alleged recurring events, and that does not involve the past tense. Two persons writing is occurring right here and right now is a fairly similar example to Chisholm's. It involves some kind of recurrence of the "same" event. It is a true statement, because right here and now two persons are writing, and it seems to be the same recurring event. Our proposal could answer this problem by stating that we are identifying a fact and not an event. In our ontology, once quantification is involved we have to go some levels up. No concrete entities are quantifiable, only abstract entities are. Thus, we have a level 2 fact which involves a numerical property that is instantiated in level 1 facts. In this case, we would have two level 1 facts that may result - for instance - from the instantiation of the property being an occurrence in two different events (in this case, one would be <being an occurrence, my writing of this article right here and now $>$ and the other <being an occurrence, his writing of his tweets right here and now $>$ ). Then, we would have a numerical property (similar to Frege's numerical property (Frege 1953: §46)) that is instantiated in two level 1 facts. This results in a level 2 fact: <numerical property two, <being an occurrence, my writing of this article right here and now $>$, $<$ being an occurrence, his writing of his tweets right here and now $>>$. The problem of simultaneous and similar events is, thus, answered: we are not dealing with a generic event, or a recurring event. We are quantifying over facts which result from the instantiation of the same property in different events. We are not identifying recurring events, we are identifying co-existing facts.

Someone could argue that this is not what Chisholm had in mind when he talked about recurrence, that he was thinking about something as the sunset which happens every day. We defend that different (but very similar events) occur on different days and, thus, what seems to be recurrence is not recurrence at all. We could perceive very similar mosquitoes, but no one would accept that they 
are recurring mosquitoes. Our idea, thus, is that events should be treated in a similar way to how we treat objects: since they are concrete they do not recur. ${ }^{20}$ There are states of affairs that involve properties that apply to events, like <universal subordination, being a sunset, being an event $>$. This is a general and atemporal state of affairs, which allows us to speak about the sunset being an event. There is also an atemporal property, like being a sunset. Since the property is universal, it allows us to speak about there being sunsets. However, they are both abstract entities and should not be confused with concrete events. There are no general events, only particular events. What is usually understood as recurrence of events is quantification over abstract entities, and not recurrence of concrete events, as mentioned above.

Chisholm claims that (17) may be resolved by his theory by paraphrasing storm into some gerundive phrase that identifies a state of affairs, and then applies the idea of concretization to explain how we may talk about coming to existence. For our theory, having an event name like storm is not a problem. Quite the contrary: we could say, in a Quinean fashion, that the fact that we have names for storms allows us to commit ontologically to events. We even have proper names for some events. We could hear a forecaster say 'Doris is coming' referring to a storm or a hurricane. This sort of linguistic facts makes the paraphrasing even harder (close to impossible). If we have to resort to some artificial paraphrasing of the proper name which does not work at all, shouldn't we take the more parsimonious way out, and accept that there are concrete events such as storms? Even though defending that having common nouns (and proper names) to refer to events could be taken as evidence that events are not reducible entities may force the matter a bit, we should ask ourselves if defending that events are reducible to states of affairs because they may be sometimes paraphrased with gerundives is not forcing the matter a lot more. In addition, we may defend that we may talk about events coming into being and being over, because they are concrete. We may talk about them as we talk about objects: we may talk about an object coming into being and being over (or dying). This is not

${ }^{20}$ We have not addressed here cases as seeing a zebra for a second time (Chisholm 1990: 424). However, we think that it could be dealt with in a similar way to this one in combination to the example found in (20). 
something we can do with states of affairs, or with facts, since both of them are abstract, and they do not concretize in any way.

Chisholm (1976: 131) argues that something like the performance of the song does not have a beginning or an end, and that is why thinking about events as concretized states of affairs is the best alternative. ${ }^{21}$ It is undeniable that the performance of the song has no beginning and no end. However, to explain this we do not need to accept a theory of concretized states of affairs. We need to recognize that, in cases as this one, we are talking about the property being a performance of the song. As a universal property, it has no beginning and no end. It is an abstract entity, completely independent of it being instantiated. When it is not instantiated it has all the properties that Chisholm recognized. When it is instantiated in an event (since it is an event property), we have as a result a temporal fact like <being the performance of the song, the performance of the song by Andrea Bocelli $>$. That fact comes into existence, when the event comes into being. That event occurs in some space-time continuously, is concrete and particular. It is that event that we may perceive, and it is that event - not the property - that may be said to be grandiose. The property, since it is here understood as a Platonic universal, is atemporal, and has no beginning or end.

We have omitted talking about causality, up to now, because it is a very complicated subject. It is strongly related to epistemology and causal explanation, and its ontological status is questionable. Faye (2010: 460), for instance, defends that it is a construct of the human being, and that "a notion like causality is a linguistic construction to speak about the actual observation with respect to past and future observations". Nevertheless, our ontology could deal with the talk about causality if it were extended to deal with time (with which it does not deal, as mentioned previously). In that case, it could be stated that some events are causally related to others, without reducing events to states of affairs. We could defend that an event $x$ is such that it causes an event $y$. That is, some events cause other events. This

\footnotetext{
${ }^{21}$ Note that we changed Chisholm's example, the performance of the symphony, to the performance of the song. This is due to the fact that a symphony involves many musicians, and thus would not turn to be a single event. A song may be performed by one musician alone, and may constitute an event.
} 
argument could be used to explain (18), if it were a sentence that dealt with current events. However, this is an issue that should be further investigated. Perception, another complicated issue, comes into account with (19). Our ontology forces us not to accept that Smith's having perceived something corresponds with a state of affairs. If we eliminate the past tense in (19), and paraphrase it to Smith sees John being robbed, we may describe perception as something that we may only experience with concrete entities. Thus, we may argue that Smith can see or perceive John being robbed because it corresponds to a concrete event. If it were a fact or a state of affairs, Smith would not have been able to see it or perceive it (for we cannot perceive the abstract). The grasping of the abstract is a different problem, but we certainly cannot perceive the abstract. However, we may perceive concrete events.

(20) allows us to recognize four alleged events: two prior that did nothing to Jones, and a third one that contributed causally to his illness (the fourth alleged event). However, none of these events exist now. Imagine, nonetheless, that Jones is still ill. This was caused by the third occurrence, and not by the others. If this were so, the explanation would follow the same path as (18), and would be causally explained. How the past events relate to the current event is something we may know first hand (we may have been with Jones at all times, and learned that it was the third occurrence that caused his being ill). That we know this by first hand allows us to talk about the events that occurred, but this does not mean that we need to reduce them to abstract entities. Knowledge and language need to be differentiated from ontology. We know that those events happened, but they are no longer here. We may talk about things that happened without the need to postulate that, since we may talk about them, they exist. We talk about historical politicians as Churchill or dead philosophers as Frege without committing ourselves to their existence: why should we commit to events that no longer exist? The fact that we may talk about what happened does not mean that we have to commit ourselves to the existence of everything about which we may speak or reason about, as we mentioned earlier. 


\section{Concluding remarks}

Chisholm (1976) presents some interesting problems for those defending an ontology that deals with events, propositions and states of affairs. He argues, and we agree, that if you want to defend an ontology that deals with those entities you need to be able to explain to what some sentences he analyzed could refer. He states that only his ontology answers these problems effectively. His ontology reduces all complex entities to states of affairs which are abstract entities which may be accepted by someone. He presents, thus, a very broad ontology of states of affairs because almost anything may be a state of affairs (or reduced to it). We have states of affairs as there being round squares, there being unicorns, there being cats, and infinite others. Some relate to concrete things, like Brutus killing Caesar, but others do not, like there being round squares. Chisholm, then, defends that both propositions and events may be reduced to states of affairs: propositions would be states of affairs that may be true or false, and events are concretizations of states of affairs. With his theory he explains the 13 cases we analyzed, and argues that he knows of no other theory that may solve the problems as effectively. We, then, stated that we thought that our ontology could solve those problems.

We defend a Platonist ontology hierachized in levels and types, which includes as basic ontological categories objects, events, properties, facts and states of affairs. Objects and events are concrete entities and pertain to level 0 . Objects are the simplest entities, and may be spatio-temporally located. Events are concrete particulars that involve objects in happenings. They are spatio-temporally continuous, and independent of our (collective) knowledge and culture. Properties, facts, and states of affairs are abstract entities. Properties are understood as Platonic universals and may be non-logical or logical. Facts involve the instantiation of a property in a concrete entity or entities. States of affairs involve the instantiation of a property in a property/ies and/or state/s of affairs. We defend that facts (such as $<$ being brown, a $\operatorname{dog}>$ or $<$ Existence $2,<$ being brown, a $\operatorname{dog}>>$ ) are temporal, particulars and non-logical; while states of affairs (such as $<$ Universal Subordination, being human, being mortal $>$ or $<$ Existence2, Diversityl $>$ ) are atemporal, general and may be non-logical or logical.

We argue that our ontology can answer to Chisholm's problems 
efficiently. We agree with him in that the first 6 can be analyzed as propositions. Since propositions are not a part of our article, we considered the last 7 (which we renumbered 14-20). We analyzed all of them and showed that our theory resolves the problems: we defended that, since events are concrete, past events do not exist and cases like (14) do not identify uniquely an event. We stated that occurring events were not a problem for our ontology. We argued that Chisholm was right in stating that something like the performance of the song does not have a beginning or an end, and defended that this was due to the fact that we are in front of an atemporal and uninstantiated property. We defended that the problem of generic events could be resolved by stating that, in cases in where recurrence seems to be involved, we are in front of facts about events and not in front of events. Finally, we defended that the fact that we may talk about nonexistent events does not make us commit to an atemporal notion of events, as neither does the fact that we may talk about historical politicians commit us to the existence of atemporal objects.

We conclude that our ontology solves the problems posed by Chisholm and that it does so in a natural way. Differentiating events, from facts and states of affairs allows us to explain many of the philosophical problems that usually arise regarding these entities, and provides analytic power to our ontology. In this particular case, it allows us to explain why it seems that we may quantify over events, and why we may talk about recurring events without using Ockham's razor. If we accept that facts may be constituted by properties, objects and/or events, many of our common talk is explained. We do not need to accept, for instance, a generic event as the sunset. We may talk about a property being a sunset that is instantiated in a concrete event such as the sunset that's happening right now. We may talk about a storm coming into being as well as we may talk about an object coming into being. We may talk about co-occurring events without assuming that they are recurring events. By approaching the problems raised by Chisholm (1976) and solving them, we showed that our proposal is a promissory one. ${ }^{22}$

Ana Clara Polakof

${ }^{22}$ This article was possible thanks to $\mathrm{CNPq}$-Brazil. 
Dept. of Philosophy Pontifícia Universidade Católica do Rio de Janeiro 22451900 Rio de Janeiro, Brazil anaclarapo@gmail.com

\section{References}

Chateaubriand, Oswaldo. 2001. Logical Forms. Part 1. Truth and Description. Campinas: Coleção CLE.

Chateaubriand, Oswaldo. 2005. Logical Forms. Part II. Logic, Language, and Knowledge. Campinas: Coleçao CLE

Chisholm, Roderick. 1976. Person and Object: A Metaphysical Study. La Salle/ Illinois: Open Court Publishing Company.

Chisholm, Roderick. 1990. Events without times: an essay in ontology. Noûs 24(3): 413-427.

Davidson, Donald. 1981a. Essays on Actions and Events. New York: Oxford University Press, 2001.

Davidson, Donald. 1981b. Eternal versus ephemeral events. In Essays on Actions and Events. New York: Oxford University Press, 2001.

Dretske, Fred. 1967. Can events move? Mind. New Series 76(304): 479-492.

Faye, Jan. 2010. Causality, contiguity, and construction. Organon F 17(4): 443-460.

Feldman, Richard and Feldman, Fred. 2015. Roderick Chisholm. In The Stanford Encyclopedia of Philosophy, ed. by Edward N. Zalta (Spring 2015 Edition). URL $=<$ http://plato.stanford.edu/archives/spr2015/entries/chisholm/> [Accessed 10/11/2015].

Frege, Gottlob. 1953. The Foundations of Arithmetic. Translated by J. L. Austin. Second Revised Edition. New York: Harper and Brothers.

Frege, Gottlob. 1964. The Basic Laws of Arithmetic. Translated and edited by Montgomery Furth. Berkeley, Los Angeles and London: University of California Press.

Honderich, Ted. 1995. The Oxford Companion to Philosophy. Oxford and New York: Oxford University Press, 2005.

Joseph, Marc. 2004. Donald Davidson. London and New York: Routledge, 2014.

Kim, Jaegwon. 1976. Events as property exemplifications. In Action Theory, ed. by M. Brand and D. Walton. Dordrecht: Reidel: 159-177.

Lemmon, E. J. 1967. Comments on D. Davidson. In The Logic of Decision and Action, ed. by N. Rescher. Pittsburgh: University of Pittsburgh Press: 96-103.

Lewis, David K. 1986. Events. In Philosophical Papers. Volume II. New York and Oxford: Oxford University Press: 241-269.

Lowe, E. J. 2001. The Possibility of Metaphysics: Substance, Identity, and Time. Oxford: Clarendon Press.

Lowe, E. J. 2006. The Four Category Ontology. New York. Oxford University Press. 
Martin, C. B. and Heil, John. 1999. The Ontological Turn. Midwest Studies in Philosophy, 23: 34-60.

Parsons, Terence. 1999. Referring to nonexistent objects. In Metaphysics: An Anthology, ed. by J. Kim and E. Sosa. Melbourne: Blackwell: 36-44.

Russell, Bertrand. 1908. Mathematical logic as based on the theory of types. American Journal of Mathematics 30(3): 222-262.

Sautter, Frank. 2010. Chateaubriand's realist conception of logic. Axiomathes 20: 357-364.

Vendler, Zeno. 1967. Facts and events. In Linguistics and Philosophy. Ithaca, NY: Cornell University Press.

Whitehead, Alfred N. and Russell, Bertrand. 1910. Principia Mathematica. Cambridge: Cambridge University Press.

Zalta, Edward. 1993. Twenty-five basic theorems in situation and world theory. Journal of Philosophical Logic 22: 385-428. 